\title{
Does Anti-Vascular Endothelial Growth Factor Treatment Delay Photoreceptor Maturation in Preterm Infants?
}

\author{
Elçin Süren, Ersan Çetinkaya*, Mehmet Fatih Küçük, \\ Musatafa Kalaycı, Muhammet Kazim Erol \\ Antalya Training and Research Hospital, Department of Ophthalmology, Antalya, Turkey \\ Email: ${ }^{\star}$ ersan_cetinkaya@hotmail.com
}

How to cite this paper: Süren, E., Çetinkaya, E., Küçük, M.F., Kalaycı, M. and Erol, M.K. (2020) Does Anti-Vascular Endothelial Growth Factor Treatment Delay Photoreceptor Maturation in Preterm Infants? Open Journal of Ophthalmology, 10, $10-20$.

https://doi.org/10.4236/ojoph.2020.101002

Received: October 17, 2019

Accepted: December 21, 2019

Published: December 24, 2019

Copyright $\odot 2020$ by author(s) and Scientific Research Publishing Inc. This work is licensed under the Creative Commons Attribution International License (CC BY 4.0).

http://creativecommons.org/licenses/by/4.0/

\begin{abstract}
Purpose: To investigate the effects of intravitreal anti-vascular endothelial growth factor (VEGF) for treatment of type-1 or aggressive posterior retinopathy of prematurity (ROP) on photoreceptor maturation in preterm infants using spectral domain optical coherence tomography (SD-OCT). Methods: A study group of 36 patients with ROP treated with anti-VEGF therapy was compared to a control group of 80 patients with ROP. All patients had undergone SD-OCT imaging at least once between 40 and 44 weeks post-menstrual age (PMA). Two imaging specialists evaluated the images for ellipsoid zone (EZ) development, cystoid macular edema (CME), sub-retinal fluid (SRF) at the fovea, and cone outer segment tips (COST). The outer nuclear layer thickness and the distance from the EZ to the central fovea was measured. Results: EZ development was observed in 18 (50\%) patients in the study group and $68(85 \%)$ in the control group ( $\mathrm{p}=$ 0.002). CME was observed in 17 (47.2\%) patients in the study group and 17 $(21.2 \%)$ in the control group. The distance between the EZ and central fovea was $868 \pm 440 \mu \mathrm{m}$ in the study group and $725 \pm 380 \mu \mathrm{m}$ in the control group $(\mathrm{p}=0.02)$. COST was not observed in any patient. Regression analysis indicated that anti-VEGF treatment and CME affect EZ development. Conclusions: Early photoreceptor maturation is correlated with preterm birth week but not ROP stage. Anti-VEGF treatment delays photoreceptor maturation in preterm infants.
\end{abstract}

\section{Keywords}

Cystoid Macular Edema, Photoreceptor, Retinopathy of Prematurity, Vascular Endothelial Growth Factor 


\section{Introduction}

Retinopathy of prematurity (ROP) is a type of proliferative vitreoretinopathy (PVR) in preterm and low birth-weight infants characterized by abnormal proliferation of retinal blood vessels [1]. Although recent advances in assisted reproductive technology (ART) have increased the rate of preterm birth, developments in neonatology have increased the odds of preterm infant survival [2]. To prevent development of permanent visual loss or blindness in infants with risk factors, it is imperative to identify the ROP risk factors and perform routine scanning to ensure early diagnosis and treatment. Despite identification of ROP risk factors, implementation of precautionary procedures, and the availability of treatment tor refraction-related visual deficiencies, the risk of lower visual acuity remains higher in preterm infants than mature infants [3].

Optical coherence tomography (OCT) is an imaging technology that provides cross-sectional images of the retina, allowing independent evaluation of ocular layers, including the retina pigment epithelium and photoreceptor cells, for diagnosis and monitoring of various diseases in adults. Most fixing and positioning problems that formerly made OCT use difficult in infants have been resolved by the introduction of portable OCT [4]. Although studies using portable spectral domain ocular coherence tomography (SD-OCT) have increased knowledge of fovea development in premature infants, it remains not fully understood. There is strong evidence that retinal layers play an important role in foveal maturation in preterm infants in a manner that affects visual development in the long term.

Studies using SD-OCT have examined foveal morphology, parafoveal retina, and structural changes with maturation [5]-[11]. These studies have revealed that even in clinically normal cases, disruptive changes in the fovea may occur. Specifically, independent of ROP existence, preterm infants may experience changes in macular structures not experienced by full-term infants, resulting in a thinner retina nerve fiber layer, flatter foveal pit, smaller foveal avascular zone diameter, and abnormal foveal photoreceptor layer [12] [13] [14].

Vascular endothelial growth factor (VEGF), an important regulator in fetal development, is released in response to the higher oxygen demand of the retinal tissue, leading to the development of blood vessels from the optic nerve to the periphery [15]. Knowledge of the key role of VEGF in ROP has encouraged researchers to explore the effect and safety of anti-VEGF drugs. Although many studies have examined the efficacy of anti-VEGF treatment, they have not been able to establish its safety. However, they have discovered that inhibition of VEGF affects regulation of angiogenesis, the functions and structures of the developing retina, and neurodevelopment of the central nervous system [11] [14] [16] [17]. Moreover, investigation of recently developed anti-VEGF drugs has shown that they inhibit some proteins expressed in photoreceptors [17]. In this study, we investigated the effect of anti-VEGF administration for treatment of type-1 or aggressive posterior ROP on photoreceptor maturation in infants. 


\section{Methods}

The study was approved by the Ethics Committee of the Antalya Training and Research Hospital and performed in accordance with the Declaration of Helsinki. Informed consent was obtained from the parents or guardians of all participating subjects prior to study participation. The medical records of 355 patients who had undergone macular SD-OCT imaging for ROP between May 2013 and October 2016 were investigated retrospectively for possible study inclusion.

The inclusion criteria were administration of intravitreal anti-VEGF treatment for ROP and macular SD-OCT imaging of both eyes at least once between gestational Week 40 and 44. The exclusion criteria were diagnosis of any anterior segmental abnormalities other than ROP or posterior-segmental abnormalities, such as congenital glaucoma, congenital cataract, or choroidal coloboma; morning glory syndrome; or oculocutaneous albinism.

Of the 355 patients examined, 116 met the inclusion criteria and none of the exclusion criteria. The control group consisted of 80 randomly selected patients who had undergone scanning for ROP and macular SD-OCT imaging at least once between gestational Week 40 and 44 .

In all ROP examinations, placement of a lid speculum was followed by careful examination of the central and peripheral fundus with scleral indentation using the RetCam digital imaging system (Clarity Medical Systems, Pleasanton, CA, USA). OCT images of the central fovea were then obtained using the iStand, a rolling floor stand option for the iVue SD-OCT system (Optovue, Fremont, USA) for scanning with a cross-line pattern. iVue obtains images of tissue with a $5-\mu \mathrm{m}$ axial resolution using an $800-\mathrm{nm}$ light source, while cross-line scanning produces one horizontal and one vertical $2 \times 6 \mathrm{~mm}$ image with 2.048 A scans and a $5-\mu \mathrm{m}$ resolution. The patients were placed in the supine position with hands and head held by a nurse. A pacifier dipped in 30\% dextrose was used to calm the infants without the use of anesthesia and lubricant eye drops (Systane, Alcon Pharmaceuticals Ltd., Barcelona, Spain) provided cornea hydration as necessary. At least three scans were performed within $10 \mathrm{~min}$ to obtain at least three images of sufficient quality, in accordance with the manufacturer's recommendations. Low-quality images were not reviewed.

After converting the SD-OCT images into digital images and communications in a medical format, two imaging specialists evaluated them for the presence of retinal layers, including ellipsoid zone (EZ) development; macular pathology, including cystoid macular edema (CME); sub-retinal fluid (SRF) at the fovea; and cone outer segment tips (COST). The outer nuclear layer thickness and the distance from the EZ to the central fovea was measured (Figure 1). The SPSS 16 program was used for statistical analysis. Within groups, non-parametric tests (the Pearson chi-square test, Mann-Whitney U test, and Kruskal-Wallis $\mathrm{H}$ test) were performed to compare the distance between the EZ and the fovea centralis and the extent of EZ development in the fovea. The Spearman's correlation test was used for correlation analysis. Values of $\mathrm{p}<0.05$ were considered significant. 


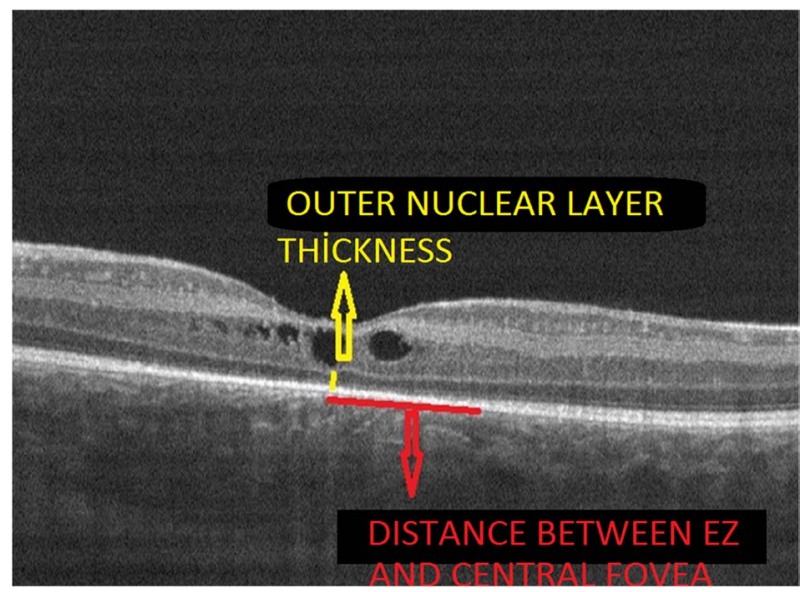

Figure 1. Shows cystoid macular edema in the inner nuclear layer. Yellow arrow shows how outer nuclear layer thickness is measured (from the outer plexiform layer to the retinal pigment epithelium) and the red arrow shows how the distance between the ellipsoid zone and the central fovea is measured (the closest point at which EZ formation can be seen to the central fovea).

\section{Results}

Of the 116 patients ( 68 females and 48 males) in the study group, 36 had been treated with anti-VEGF therapy for ROP. These 36 patients were examined further and their findings compared to those of the 80 patients ( 35 males and 45 females) in the control group (Table 1 and Table 2). The mean gestational age of all patients was $30.1 \pm 2.1$ weeks, the mean birth weight $1236.7 \pm 401 \mathrm{gm}$, and the mean age during imaging was $41.4 \pm 1.8$ weeks post-menstrual age (PMA). The mean gestational age was $29.6 \pm 2.5$ weeks in the study group and $30.3 \pm 3.1$ weeks in the control group. The mean age during imaging was $42.1 \pm 1.8$ weeks PMA in the study group and $41.1 \pm 1.8$ weeks in the control group. The mean birth weight was $1125.2 \pm 314.5 \mathrm{gm}$ in the study group and $1287.4 \pm 464.7 \mathrm{gm}$ in the control group. In the study group 32 patients had been treated with intravitreal (IV) ranibizumab and 4 with IV bevacizumab (Table 1). In the control group 12 patients had been treated for ROP with argon laser photocoagulation (Table 2). In the study group, 14 (38.9\%) patients exhibited stage 2 ROP and 22 (61.1\%) stage 3 ROP. Plus disease was observed in all 36 patients in the study group. In the control group, 40 (50\%) patients exhibited no ROP, 18 (22.5\%) stage 1 ROP, 16 (20\%) stage 2 ROP, and 6 (7.5\%) stage 3 ROP (Table 3). In the control group, $62(77.5 \%)$ patients exhibited no plus disease, $12(15 \%)$ pre-plus disease, and $6(7.5 \%)$ plus disease (Table 4 ).

Figures 1-3 show the extent and characteristics of EZ development. EZ development was observed in 18 (50\%) patients in the study group and $68(85 \%)$ in the control group, a statistically significant difference $(\mathrm{p}=0.002)$. CME was observed in 17 (47.2\%) patients in the study group and 17 (21.2\%) in the control group, a statistically significant difference $(\mathrm{p}=0.02)$. COST was not observed in any patients (Table 5). Regression analysis indicated that anti-VEGF treatment and CME affect EZ development. 
Table 1. Demographic characteristics.

\begin{tabular}{cccc}
\hline Characteristic & $\begin{array}{c}\text { Study group } \\
(\mathbf{n}=\mathbf{3 6})\end{array}$ & $\begin{array}{c}\text { Control group } \\
(\mathbf{n}=\mathbf{8 0})\end{array}$ & P value \\
\hline Gestational age (weeks) & $29.6 \pm 2.5$ & $30.3 \pm 3.1$ & 0.678 \\
Mean gestational age during imaging (weeks) & $42.1 \pm 1.8$ & $41.1 \pm 1.8$ & 0.470 \\
Birth weight (gm) & $1125.2 \pm 314.5$ & $1287 \pm 464.7$ & 0.124 \\
Sex (n and \% males) & $18(50 \%)$ & $35(43.7 \%)$ & 0.236 \\
\hline
\end{tabular}

Table 2. Treatment modalities.

\begin{tabular}{ccc}
\hline Treatment & Study group $(\mathrm{n}=36)$ & Control group $(\mathrm{n}=\mathbf{8 0})$ \\
\hline Intravitreal ranibizumab (n) & 32 & 0 \\
Intravitreal bevacizumab (n) & 4 & 0 \\
Laser photocoagulation (n) & 0 & 12 \\
\hline
\end{tabular}

Table 3. ROP Stage.

\begin{tabular}{ccc}
\hline Maximum ROP stage & Study group $(\mathbf{n}=\mathbf{3 6})$ & Control group $(\mathbf{n}=\mathbf{8 0})$ \\
\hline No ROP $(\mathrm{n}, \%)$ & 0 & $40(50.0 \%)$ \\
Stage 1 (n, \%) & 0 & $18(22.5 \%)$ \\
Stage 2 (n, \%) & $14(38.9 \%)$ & $16(20.0 \%)$ \\
Stage 3 (n, \%) & $22(61.1 \%)$ & $6(7.5 \%)$ \\
\hline
\end{tabular}

Table 4. Plus disease status.

\begin{tabular}{ccc}
\hline Status & Study group $(\mathbf{n}=\mathbf{3 6})$ & Control group $(\mathbf{n}=\mathbf{8 0})$ \\
\hline Plus disease negative $(\mathrm{n}, \%)$ & 0 & $62(77.5 \%)$ \\
Preplus disease (n, \%) & 0 & $12(15.0 \%)$ \\
Plus disease (n, \%) & $36(100 \%)$ & $6(7.5 \%)$ \\
\hline
\end{tabular}

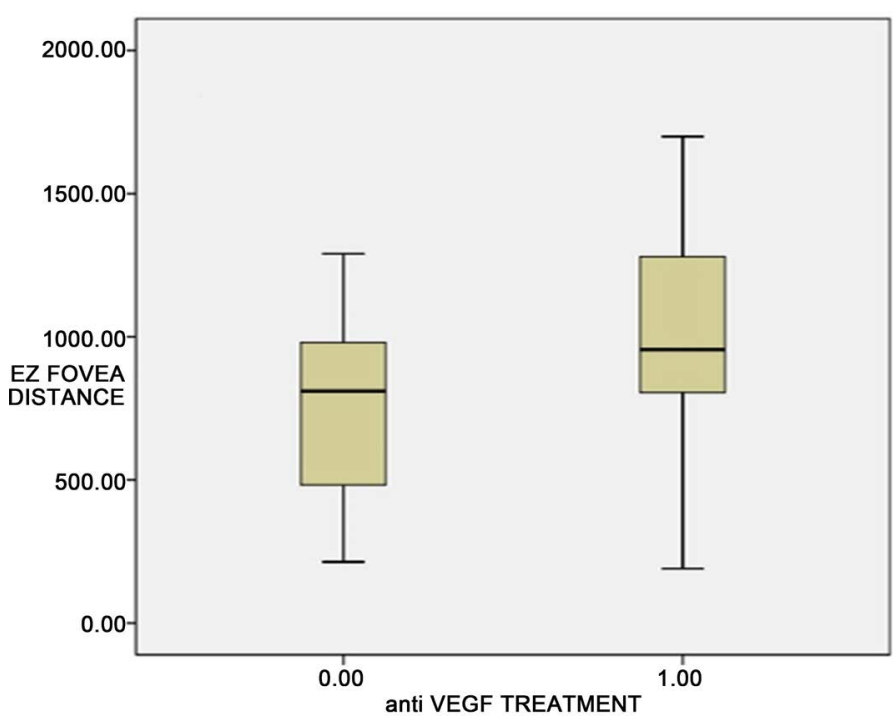

Figure 2. Comparison of the distance between the ellipsoid zone and the fovea in the study and control groups. 


\begin{tabular}{cccc}
\hline & SE & WALD & P value \\
\hline ROP STAGE & 0.736 & 2.540 & 0.111 \\
ANTI VEGF TREATMENT & 1.853 & 4.679 & 0.031 \\
PRESENCE OF CME & 0.765 & 5.597 & 0.018 \\
BİRTH WEEK & 0.189 & 0.038 & 0.844 \\
BİRTH WEİGHT & 0.01 & 0.044 & 0.833 \\
\hline
\end{tabular}

Figure 3. Findings of regression analysis of the factors that affect ellipsoid zone formation.

Table 5. OCT parameters.

\begin{tabular}{cccc}
\hline Parameter & Study group $(\mathrm{n}=36)$ & Control group $(\mathrm{n}=80)$ & P value \\
\hline Ellipsoid zone development $(\mathrm{n}, \%)$ & $18(50 \%)$ & $68(85 \%)$ & 0.002 \\
Cystoid macular edema $(\mathrm{n}, \%)$ & $17(47.2 \%)$ & $17(21.2 \%)$ & 0.02 \\
Cone outer segment tips $(\mathrm{n}, \%)$ & 0 & 0 & \\
Outer nuclear layer thickness $(\mu \mathrm{m})$ & $47.7 \pm 21$ & $56.9 \pm 12$ & 0.04 \\
\hline
\end{tabular}

\section{Discussion}

Histological studies of fetal eye development began in 1964 with observation of the persistence of inner retina layers in the fovea centralis, abnormal foveal vascular formation in fetal eyes, and alterations in the width and length of the photoreceptors over time [18]. The foveal maturation process was defined as the centrifugal migration of inner retinal layers and centripetal migration of cone cells. Research into the anatomical development of the premature fovea identified formation of the parafoveal avascular zone during mid-gestation; similar development during mid-gestation among ROP patients; and the influence of various factors on the foveal maturation process, such as prematurity, vessel formation, foveal tissue elasticity, intra-ocular pressure (OP), retinal stretching, hypoxia, and VEGF, some of which play a synergic role [19] [20].

The development of inner and outer retinal layers at the fovea may progress independently. This dynamic process of foveal maturation and specialization can be studied in vivo in premature infants with SD-OCT imaging. Bach and Seefelder first described the development of the human fovea and described foveal photoreceptor immaturity [21]. Regarding the latter, Moldano et al. showed that compared to adults, immature infants exhibited persistence of the inner retina layers, and while the inner layers exhibited centrifugal development, the outer layers exhibited centripetal development [7]. Hendrickson et al. confirmed these findings with histological studies and showed that the foveal outer nuclear layer in postnatal infants progressively thickened as cone packing and elongation of the inner segment (IS) and outer segment (OS) occurred [22].

The inner segment EZ is an array of longitudinal-oriented mitochondria that appears as a hyper-reflective band on OCT. Initially, IS and OS are observed as the hyper-reflective retina pigment epithelium (RPE) thickens at approximately 
33 to 36 weeks post-menstrual age (PMA) [23] [24] [25]. Subsequently, the EZ is separated from the similarly reflective RPE by elongation of the hyper-reflective OS in the periphery at approximately 37 to 39 weeks PMA [24] [25] [26] [27]. In a prospective study, Jayadev et al. first identified the IS/OS band on the RPE at 33 weeks PMA as a barely identifiable reflective band that subsequently reached the center from the periphery during thickening at 43 - 48 weeks PMA and observed that the impact of the EZ on visual acuity was significant [28].

Previous research has demonstrated that development of photoreceptors plays great importance in visual function and visual acuity and that delayed development may affect vision. In accordance, our investigation of EZ formation and photoreceptor maturation in infants treated with anti-VEGF for ROP revealed a significant delay in EZ development at 40 - 44 weeks PMA. Specifically, we observed EZ development in only 18 of 36 infants (50\%) treated with anti-VEGF (the study group) compared to 68 of 80 (85\%) not treated with anti-VEGF (the control group).

Our findings also confirmed Vajzovic et al.'s identification of a delay in photoreceptor maturation in preterm infants compared to full-term infants, and that the delay was greater in infants with macular edema [14]. In the same study, Vajzovic et al. also observed EZ formation at the foveal center in 47\% (22/47) of the term infants but only $14 \%(9 / 64)$ of the very preterm infants $(\mathrm{p}<0.001)$ whom they imaged. Moreover, they found among those infants without EZ development at the fovea a greater mean distance between the EZ and the foveal center in very preterm compared to term infants $(\mathrm{p}=0.01)$, further indicating a delay in photoreceptor migration.

Saint-Geniez had previously shown that the endogenic presence of vascular endothelial growth factor plays a crucial role in the development of normal photoreceptors [29]. Research conducted on animals by Avci et al. showed that VEGF inhibition increased apoptotic activity in photoreceptors [30]. Although most studies of anti-VEGF treatment found no neurological adverse effects [31] [32], Morin et al. recently indicated anti-VEGF treatment in preterm infants is associated with poor neurodevelopmental outcomes [33]. Considering the neuroprotective effect of VEGF, it can be concluded that anti-VEGF treatment delays the maturation of the developing photoreceptor cell layer, a conclusion supported by our finding that anti-VEGF treatment may delay photoreceptor maturation.

Supporting Vajzovic et al.'s finding that only prematurity and CME delay EZ formation [14], we showed that CME may delay photoreceptor maturation. In contrast to their study, we observed that prematurity appears not to delay photoreceptor maturation. However, this difference may be attributed to the inclusion of term infants in their study but not our study.

Rothman et al. observed that studies using OCT have increased understanding of normal eye development and identified several potential biomarkers of brain abnormalities and poorer neurodevelopment [34]. Their observation indicates 
that elucidation of morphology using SD-OCT could assist in identifying the predictors of future visual acuity. More specifically, gaining better understanding of preterm retinal substructures and their association with brain anatomy will elucidate the pathway by which local retinal anatomic changes impact and predict subnormal vision and central nervous system (CNS) function. By identifying the age-appropriate stages of normal neonatal retinal development and maturation, researchers can identify changes associated with not only ROP but also prematurity. These include delayed photoreceptor development, CME, enlarged optic nerve cup-to-disc (C/D) ratio, and thin retinal nerve fiber layer (RNFL).

Our study indicates that anti-VEGF treatment may delay photoreceptor maturation in premature infants. Further investigation is needed to determine the effects of this delay on visual functioning. These anatomic abnormalities have been linked to abnormalities in brain anatomy and neurodevelopment. As the eye and brain continue to develop well after term birth, it is highly likely that further research in full-term infants may find similar relationships between eye microanatomy and neurodevelopment and CNS disease.

All procedures performed in studies involving human participants were in accordance with the ethical standards of the institutional and/or national research committee and with the 1964 Helsinki declaration and its later amendments or comparable ethical standards.

\section{Acknowledgements}

The study was conducted at Department of Ophthalmology, Antalya Training and Research Hospital, Antalya, Turkey. This study was presented at EVRS 2018 in Praque, which has not been published in any journal before. This study has been recognized as a medical specialty thesis in ophthalmology. We declare that there is no financial support or relationships that may pose a conflict of interest. Finally, there is no conflict of interest in connection with this submitted article, and the manuscript has been read and approved by all the authors.

\section{Conflicts of Interest}

The authors declare no conflicts of interest regarding the publication of this paper.

\section{References}

[1] Dobson, V., Quinn, G.E., Summers, C.G., Hardy, R.G. and Tung, B. (2006) Visual Acuity at 10 Years in Cryotherapy for Retinopathy of Prematurity (CRYO-ROP) Study Eyes: Effect of Retinal Residua of Retinopathy of Prematurity. Archives of Ophthalmology, 124, 199-202. https://doi.org/10.1001/archopht.124.2.199

[2] Campbell, P.B., Bull, M.J., Ellis, F.D., Bryson, C.Q., Lemons, J.A. and Schreiner, R. (1983) Incidence of Retinopathy of Prematurity in a Tertiary Newborn Intensive Care Unit. Archives of Ophthalmology, 101, 1686-1688. https://doi.org/10.1001/archopht.1983.01040020688003 
[3] Bowl, W., Lorenz, B., Stieger, K., Schweinfurth, S., Holve, K., Friedburg, C. and Andrassi-Darida, M. (2016) Correlation of Central Visual Function and ROP Risk Factors in Prematures with and without Acute ROP at the Age of 6-13 Years: The Giessen Long-Term ROP Study. British Journal of Ophthalmology, 100, 1238-1244. https://doi.org/10.1136/bjophthalmol-2015-307855

[4] Maldonado, R.S. and Toth, C.A. (2013) Optical Coherence Tomography in Retinopathy of Prematurity: Looking beyond the Vessels. Clinics in Perinatology, 40, 271-296. https://doi.org/10.1016/j.clp.2013.02.007

[5] Maldonado, R.S., O’Connell, R., Ascher, S.B., Sarin, N., Freedman, S.F., Wallace, D.K., Chiu, S.J., Farsiu, S., Cotton, M. and Toth, C.A. (2012) Spectral Domain Optical Coherence Tomographic Assessment of Severity of Cystoid Macular Edema in Retinopathy of Prematurity. Archives of Ophthalmology, 130, 569-578.

https://doi.org/10.1001/archopthalmol.2011.1846

[6] Maldonado, R.S., Freedman, S.F., Cotton, C.M., Ferranti, J.M. and Toth, C.A. (2011) Reversible Retinal Edema in an Infant with Neonatal Hemochromatosis and Liver Failure. Journal of AAPOS, 15, 91-93. https://doi.org/10.1016/j.jaapos.2010.11.016

[7] Maldonado, R.S., O’Connell, R.V., Sarin, N., Freedman, S.F., Wallace, D.K., Cotton, C.M., Winter, K.P., Stinnett, S., Chiu, S.J., Izatt, J.A., Farsiu, S. and Toth, C.A. (2011) Dynamics of Human Foveal Development after Premature Birth. Ophthalmology, 118, 2315-2325. https://doi.org/10.1016/j.ophtha.2011.05.028

[8] Lee, A.C., Maldonado, R.S., Sarin, N., O’Connell, R.V., Wallace, D.K., Freedman, S.F., Cotton, M. and Toth, C.A. (2011) Macular Features from Spectral-Domain Optical Coherence Tomography as an Adjunct to Indirect Ophthalmoscopy in Retinopathy of Prematurity. Retina, 31, 1470-1482. https://doi.org/10.1097/IAE.0b013e31821dfa6d

[9] Dubis, A.M., Subramaniam, C.D., Godara, P., Carroll, J. and Costakos, D.M. (2013) Subclinical Macular Findings in Infants Screened for Retinopathy of Prematurity with Spectral-Domain Optical Coherence Tomography. Ophthalmology, 120, 1665-1671. https://doi.org/10.1016/j.ophtha.2013.01.028

[10] Erol, M.K., Ozdemir, O., Turgut Coban, D., Bilgin, A.B., Dogan, B., Sogutlu Sari, E. and Toslak, D. (2004) Macular Findings Obtained by Spectral Domain Optical Coherence Tomography in Retinopathy of Prematurity. Journal of Ophthalmology, 2014, Article ID: 468653. https://doi.org/10.1155/2014/468653

[11] Erol, M.K., Coban, D.T., Özdemir, Ö., Tunay, Z.Ö., Bilgin, A.B. and Dogan, B. (2015) Spectral-Domain OCT Analyses of Macular Changes after Ranibizumab Therapy for Type 1 Retinopathy of Prematurity. Journal of Pediatric Ophthalmology and Strabismus, 52, 152-158. https://doi.org/10.3928/01913913-20150326-12

[12] Fieß, A., Christian, L., Janz, J., Kölb-Keerl, R., Knuf, M., Kirchhof, B., Muether, P.S., Bauer, J. and Prematurity Eye Study Group (2017) Functional Analysis and Associated Factors of the Peripapillary Retinal Nerve Fibre Layer in Former Preterm and Full-Term Infants. British Journal of Ophthalmology, 101, 1405-1411. https://doi.org/10.1136/bjophthalmol-2016-309622

[13] Hammer, D.X., Iftimia, N.V., Ferguson, R.D., Bigelow, C.E., Ustun, T.E., Barnaby, A.M. and Fulton, A.B. (2008) Foveal Fine Structure in Retinopathy of Prematurity: An Adaptive Optics Fourier Domain Optical Coherence Tomography Study. Investigative Ophthalmology \& Visual Science, 49, 2061-2070.

https://doi.org/10.1167/iovs.07-1228

[14] Vajzovic, L., Rothman, A.L., Tran-Viet, D., Cabrera, M.T., Freedman, S.F. and Toth, 
C.A. (2015) Delay in Retinal Photoreceptor Development in Very Preterm Compared to Term Infants. Investigative Ophthalmology \& Visual Science, 56, 908-913. https://doi.org/10.1167/iovs.14-16021

[15] Lashkari, K., Hirose, T., Yazdany, J., McMeel, J.W., Kazlauskas, A. and Rahimi, N. (2000) Vascular Endothelial Growth Factor and Hepatocyte Growth Factor Levels Are Differentially Elevated in Patients with Advanced Retinopathy of Prematurity. The American Journal of Pathology, 156, 1337-1344.

https://doi.org/10.1016/S0002-9440(10)65004-3

[16] Sankar, M.J., Sankar, J., Mehta, M., Bhat, V. and Srinivasan, R. (2017) Anti-Vascular Endothelial Growth Factor (VEGF) Drugs for Treatment of Retinopathy of Prematurity. Cochrane Database of Systematic Reviews, 2, CD009734. https://doi.org/10.1002/14651858.CD009734.pub2

[17] Shen, W., Yau, B., Lee, S.R., Zhu, L., Yam, M. and Gillies, M.C. (2017) Effects of Ranibizumab and Aflibercept on Human Müller Cells and Photoreceptors under Stress Conditions. International Journal of Molecular Sciences, 18, 533. https://doi.org/10.3390/ijms18030533

[18] Mann, I. (1964) The Development of the Human Eye. 3rd Edition, Grune and Stratton, New York.

[19] Hendrickson, A.E. and Yuodelis, C. (1984) The Morphological Development of the Human Fovea. Ophthalmology, 91, 603-612. https://doi.org/10.1016/S0161-6420(84)34247-6

[20] Yuodelis, C. and Hendrickson, A.E. (1986) A Qualitative and Quantitative Analysis of the Human Fovea during Development. Vision Research, 26, 847-855. https://doi.org/10.1016/0042-6989(86)90143-4

[21] Bach, L.S.R. (1914) Atlas zur Entwicklungsgeschichte des Menschlichen Auges. Leipzig.

[22] Hendrickson, A., Possin, D., Vajzovic, L. and Toth, C.A. (2012) Histologic Development of the Human Fovea from Midgestation to Maturity. American Journal of Ophthalmology, 154, 767-778. https://doi.org/10.1016/j.ajo.2012.05.007

[23] Vajzovic, L., Hendrickson, A.E., O’Connell, R.V., Clark, L.A., Tran-Viet, D., Possin, D., Chiu, S.J., Farsiu, S. and Toth, C.A. (2012) Maturation of the Human Fovea: Correlation of Spectral-Domain Optical Coherence Tomography Findings with Histology. American Journal of Ophthalmology, 154, 779-789. https://doi.org/10.1016/j.ajo.2012.05.004

[24] Maheshwary, A.S., Oster, S.F., Yuson, R.M., Cheng, L., Mojana, F. and Freeman, W.R. (2010) The Association between Percent Disruption of the Photoreceptor Inner Segment-Outer Segment Junction and Visual Acuity in Diabetic Macular Edema. American Journal of Ophthalmology, 150, 63-67. https://doi.org/10.1016/j.ajo.2010.01.039

[25] Ota, M., et al. (2008) Integrity of Foveal Photoreceptor Layer in Central Retinal Vein Occlusion. Retina, 28, 1502-1508. https://doi.org/10.1097/IAE.0b013e3181840b3c

[26] Vinekar, A., Mangalesh, S., Jayadev, C., Maldonado, R.S., Bauer, N. and Toth, C.A. (2015) Retinal Imaging of Infants on Spectral Domain Optical Coherence Tomography. BioMed Research International, 2015, Article ID: 782420. https://doi.org/10.1155/2015/782420

[27] Vinekar, A., Avadhani, K., Sivakumar, M., Mahendradas, P., Kurian, M., Braganza, S., Shetty, R. and Shetty, B.K. (2011) Understanding Clinically Undetected Macular Changes in Early Retinopathy of Prematurity on Spectral Domain Optical Cohe- 
rence Tomography. Investigative Ophthalmology \& Visual Science, 52, 5183-5188. https://doi.org/10.1167/iovs.10-7155

[28] Jayadev, C., Vinekar, A., Mangalesh, S., Kummelil, M.K., Kumar, A.K., Kemmanu, V., Sivakumar, M., Mahendradas, P., Avadhani, K., Bauer, N., Webers, C.A. and Shetty, B. (2016) Foveal Layer Morphology Detected on Spectral Domain Optical Coherence Tomography and Its Correlation with Visual Acuity in Asian Indian Premature Infants in Their First Year of Life. Current Eye Research, 42, 789-795. https://doi.org/10.1080/02713683.2016.1236964

[29] Saint-Geniez, M., Maharaj, A.S., Walshe, T.E., Tucker, B.A., Sekiyama, E., Kurihara, T., Darland, D.C., Young, M.J. and D'Amore, P.A. (2008) Endogenous VEGF Is Required for Visual Function: Evidence for Survival Role on Muller Cells and Photoreceptors. PLoS ONE, 3, e3554. https://doi.org/10.1371/journal.pone.0003554

[30] Avci, B., Avci, R., Inan, U.U. and Kaderli, B. (2009) Comparative Evaluation of Apoptotic Activity in Photoreceptor Cells after Intravitreal Injection of Bevacizumab and Pegaptanib Sodium in Rabbits. Investigative Ophthalmology \& Visual Science, 50, 3438-3446. https://doi.org/10.1167/iovs.08-2871

[31] Kana, H., Mayet, I., Soma, D., Dawood Alli, H. and Biddulph, S. (2017) The Efficacy of Intravitreal Antivascular Endothelial Growth Factor as Primary Treatment of Retinopathy of Prematurity: Experience from a Tertiary Hospital. South African Medical Journal, 107, 215-218. https://doi.org/10.7196/SAMJ.2017.v107i3.11080

[32] Martínez-García, S.M., Hernández-Da Mota, S.E., Rubio-Rangel, A., Rojas-Flores, I., Vieyra-López, M.E., Martínez-Castellanos, M.A., Zavala-Martínez, M.T. and Gómez García, A. (2017) Neurodevelopment in Patients with Retinopathy of Prematurity Treated with Intravitreal Bevacizumab. Case Series. Cirugía y Cirujanos, 85, 478-484. https://doi.org/10.1016/j.circen.2018.01.009

[33] Morin, J., Luu, T.M., Superstein, R., Ospina, L.H., Lefebvre, F., Simard, M.N., Shah, V., Shah, P.S., Kelly, E.N. and Canadian Neonatal Network and the Canadian Neonatal Follow-Up Network Investigators (2016) Neurodevelopmental Outcomes Following Bevacizumab Injections for Retinopathy of Prematurity. Pediatrics, 37, e20153218. https://doi.org/10.1542/peds.2015-3218

[34] Rothman, A.L., Tran-Viet, D., Gustafson, K.E., Goldstein, R.F., Maguire, M.G., Tai, V., Sarin, N., Tong, A.Y., Huang, J., Kupper, L., Cotton, C.M., Freedman, S.F. and Toth, C.A. (2015) Poorer Neurodevelopmental Outcomes Associated with Cystoid Macular Edema Identified in Preterm Infants in the Intensive Care Nursery. Ophthalmology, 122, 610-619. https://doi.org/10.1016/j.ophtha.2014.09.022 